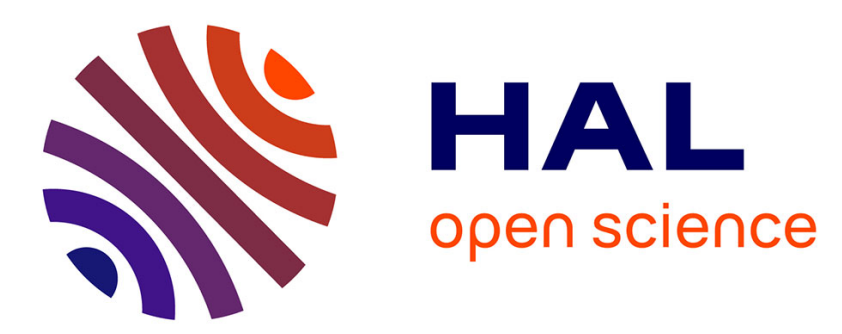

\title{
Plasmonic inverse rib waveguiding for tight confinement and smooth interface definition
}

Henri Benisty, Mondher Besbes

\section{To cite this version:}

Henri Benisty, Mondher Besbes. Plasmonic inverse rib waveguiding for tight confinement and smooth interface definition. Journal of Applied Physics, 2010, 108, pp.063108. 10.1063/1.3478746 . hal00546215

\section{HAL Id: hal-00546215 \\ https://hal-iogs.archives-ouvertes.fr/hal-00546215}

Submitted on 7 Dec 2015

HAL is a multi-disciplinary open access archive for the deposit and dissemination of scientific research documents, whether they are published or not. The documents may come from teaching and research institutions in France or abroad, or from public or private research centers.
L'archive ouverte pluridisciplinaire HAL, est destinée au dépôt et à la diffusion de documents scientifiques de niveau recherche, publiés ou non, émanant des établissements d'enseignement et de recherche français ou étrangers, des laboratoires publics ou privés. 


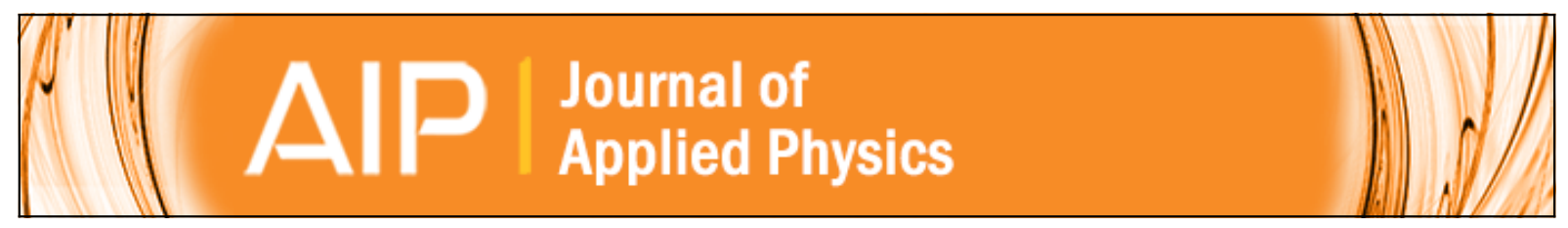

\section{Plasmonic inverse rib waveguiding for tight confinement and smooth interface definition}

$\mathrm{H}$. Benisty and M. Besbes

Citation: Journal of Applied Physics 108, 063108 (2010); doi: 10.1063/1.3478746

View online: http://dx.doi.org/10.1063/1.3478746

View Table of Contents: http://scitation.aip.org/content/aip/journal/jap/108/6?ver=pdfcov

Published by the AIP Publishing

\section{Articles you may be interested in}

Quantum-dot based nanothermometry in optical plasmonic recording media

Appl. Phys. Lett. 105, 181110 (2014); 10.1063/1.4901258

Terahertz beam focusing based on plasmonic waveguide scattering

Appl. Phys. Lett. 101, 151116 (2012); 10.1063/1.4759042

Single-mode waveguiding in bundles of self-assembled semiconductor nanowires

Appl. Phys. Lett. 97, 221915 (2010); 10.1063/1.3524219

Wideband Y-splitter and aperture-assisted coupler based on sub-diffraction confined plasmonic slot waveguides Appl. Phys. Lett. 96, 131106 (2010); 10.1063/1.3374454

The gain effect in a magnetic plasmon waveguide

Appl. Phys. Lett. 96, 113103 (2010); 10.1063/1.3365179
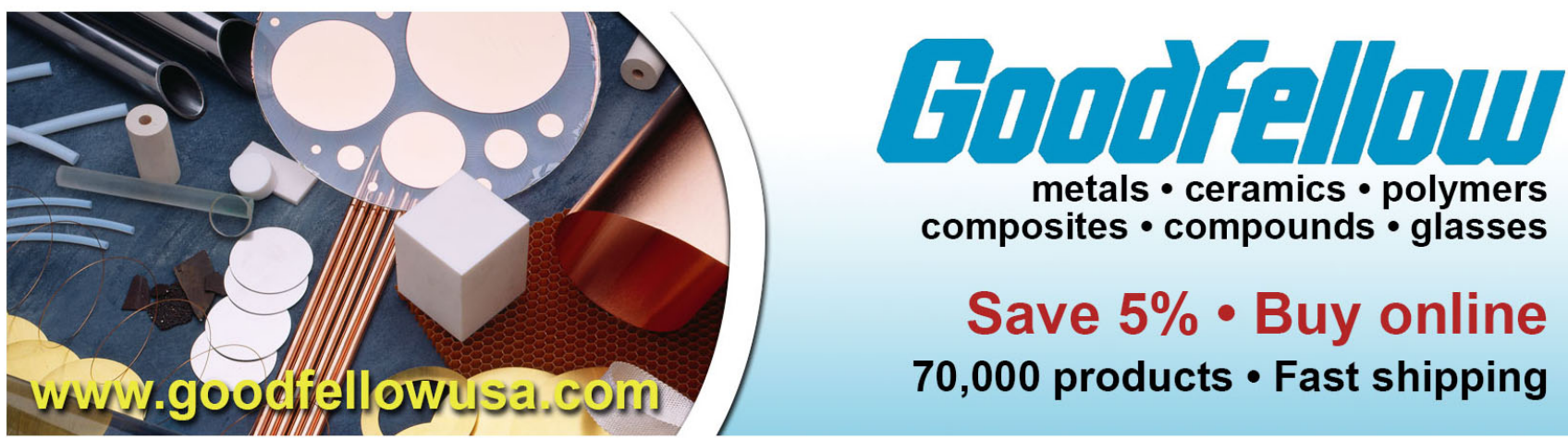


\title{
Plasmonic inverse rib waveguiding for tight confinement and smooth interface definition
}

\author{
$\mathrm{H}$. Benisty ${ }^{\mathrm{a})}$ and M. Besbes \\ Laboratoire Charles Fabry de l'Institut d'Optique, CNRS, Univ. Paris-Sud, Campus Polytechnique, RD 128, \\ 91127 Palaiseau Cedex, France
}

(Received 16 April 2010; accepted 19 July 2010; published online 24 September 2010)

\begin{abstract}
A plasmonic inverse rib optical waveguide geometry is proposed and investigated, inspired by the recent CdS-nanorod-on-silver plasmonic laser. The proposed technology is suitable for large scale fabrication. It only uses a single wet resist development and several coatings onto a flat metal surface to define the waveguide geometry. It thus relieves the need to etch or lift-off a noble metal. High-index sol-gel inverse ribs are privileged candidates for the tightest confinement. We investigate and explain the guidance mostly for the case of $\mathrm{Au}$ and the wavelengths around $\lambda$ $=633 \mathrm{~nm}$. We get spot sizes down to $\sim 25 \times 60 \mathrm{~nm}^{2}$. We notably describe how easily the tight confinement is granted and the reasons why only a single critical step defines the modal geometry. We finally detail how the classical building-blocks of integrated optics such as distributed reflectors and couplers can be made within the very same approach and integrated into devices for which losses are described. (C) 2010 American Institute of Physics. [doi:10.1063/1.3478746]
\end{abstract}

\section{INTRODUCTION}

The large efforts in plasmonic research in the last decade $^{1,2}$ ambition to merge the successful concepts of integrated optics with the miniaturizing potential of plasmonics. As shown in the recent example of the CdS-nanorod-onsilver plasmonic laser, ${ }^{3}$ not only can plasmonic successfully result in miniature confinement but this confinement further brings pure photonic advantages such as a large Purcell effect for emitters placed in the enhanced modal field. Of course, plasmonic losses will prevent complex cascaded functions to be integrated to a large scale but a number of clever "everything-on-a-chip" approaches, which involve photonics, should gain a profit from plasmonic devices.

The founding element of integrated optics is the channel waveguide. At the turn of the millennium, after a quasiabsence of studies on rib-type plasmonic geometries, Berini's papers ${ }^{4,5}$ set a milestone, soon followed by a string of investigations ${ }^{6-19}$ up to recent work on plasmons with gain materials. ${ }^{20}$ The past decade has witnessed the discovery of the many subtle effects which arise when the well known TM-polarized two-dimensional (2D) surface plasmon of noble metals is constrained by boundary conditions of a rib or a channel, with a special role of corners for instance. ${ }^{9,10,15}$ The case of two curved metallic surface forming a confined channel surface polaritons waveguide geometry was recently discussed, lending itself to an analytical approach. ${ }^{21}$ Practically, shaping tiny gold or silver ridges is routine but leaves some residual roughness because of factors difficult to master such as the weak adhesion of the metal (a thin layer) to its substrate. The alternative of the dielectric load onto flat $\mathrm{Au}$ or $\mathrm{Ag}$ is very promising in this respect, ${ }^{7,8,11}$ but the adhesion of a long rib of sputtered oxide or silicon to the underlying Au layer is not very robust either. In comparison with main-

${ }^{a)}$ Electronic mail: henry.benisty@ institutoptique.fr. stream microelectronics silicon technologies, it is somewhat more challenging to define very narrow wires on such gold layers.

We were prompted by this possible difficulty (admittedly technology-dependent) and by the desire to find the simplest method to get such plasmonic waveguides. The result is the present proposal, termed a plasmonic inverse rib optical waveguide (PIROW) geometry. It makes use of the surface plasmons and of an inverse rib shaped from materials of two different indices, a high index rib forming a small gap of low index with the metal, a geometry inspired by the CdSnanorod-on-silver plasmonic laser. ${ }^{3}$ It can also be seen as a modified two-cylinders channel waveguide of Ref. 21 with a high index dielectric load replacing the top cylinder and a vanishing curvature of the bottom cylinder. Our proposal has several merits to our eyes. We particularly describe its very tight confinement properties. We assess its large potential as part of a general strategy to implement low-cost large scale functional plasmonics, with minimal critical elaboration steps and a large flexibility. Several key functions of integrated optics can straightforwardly be achieved within our technological scheme.

The inverse rib geometry is a minority option in traditional "dielectric" integration and it is thus scarcely documented. To our knowledge, none of the few dozens of papers of the last decade that relate to the plasmonic rib geometry has alluded to an inverse rib geometry as a technological option. The outline of the paper is as follows. It first presents the geometry of interest. It then describes its photonic behavior for indices known to be available for waveguide-grade deposition techniques, notably titanium alkoxyde based solgel systems with possibly $n \sim 2.0,{ }^{22-24}$ taking the tip width as a relevant parameter. The limits imposed by lower indices are exemplified by indices of 1.65 typical of poly $(\mathrm{N}$ vinylcarbazole) (PVK) or butyl-cyclo-benzene (BCB). The results are discussed in order to establish the merits of the 

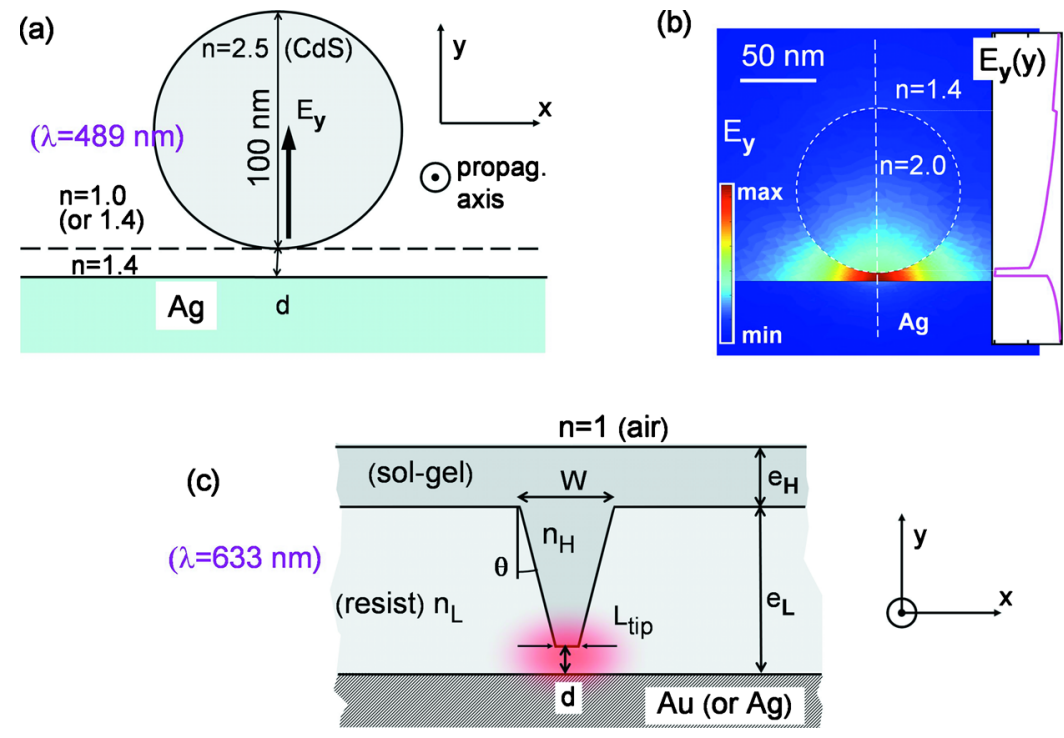

FIG. 1. (Color online) (a) Schematic of the plasmon laser nanorod configuration. Air lies above the dashed line, not being an essential ingredient though; (b) simulated $E_{y}$ field for a simplified situation and $n_{H}=2.0$; (c) the PIROW inverse rib geometry (with $\mathrm{Au}$ and sol-gel high index), with mode localization outlined by the indicative shaded spot. approach: the reasons of the tight confinement, the large tolerance to the "air side" (or top side) of the inverse rib shape, the good quality of the embedded sidewall interfaces. We subsequently give a quantitative account of the effect of gap width, and of frequency, from red to near infrared, in relation with losses. The final part of the paper discusses which patterns can be lithographically defined to straightforwardly implement a decent set of photonic devices, such as distributed Bragg reflectors (DBRs) (for lasers), outcouplers, splitters. The constrains induced by losses on Bragg reflector design are given.

\section{FROM NANOROD TO INVERSE RIB PLASMONIC WAVEGUIDING}

The operation of the plasmon laser of ${ }^{3}$ exploits a tight confinement in a very thin low index layer $\left(\mathrm{MgF}_{2}, n_{L} \sim 1.4\right)$ lying between a flat silver surface (dielectric constant $\varepsilon_{\text {metal }}$ ) and the convex bottom surface of a high index $\mathrm{CdS}$ nanorod of diameter $100 \mathrm{~nm} \sim \lambda / 2 n$ for an index $n_{H} \sim 2.5$ [Fig. 1(a)]. The predominant $E_{y}$ component of the resulting mode is confined laterally to much less than the rod diameter: it is about $40 \mathrm{~nm}$ wide in the $\mathrm{MgF}_{2}$ gap. Vertically, $E_{y}$ in this tiny gap is nearly constant on the symmetry axis, it decays quickly in the silver half-space but also quite quickly in the nanorod itself. This feature is distinctly different from the profile that would result from a perturbative hybridization of a surface plasmon at a flat $\mathrm{Au} / \mathrm{MgF}_{2}$ air system with the fundamental guided mode of the nanorod in air.

Hence the success of this strategy can be analyzed as the capability of this gap geometry to enforce a propagation constant such that the mode effective index $n_{\text {eff }}$ becomes considerably higher than that of the highest-effective-index guided mode of the high index dielectric guide alone.

This can be denoted $n_{\text {eff,diel }}<n_{\text {eff }} \# n_{H}$, the last comparison denoted \# meaning a very approximate vicinity but of variable sign. Then, see Fig. 1(a), decay is ensured vertically in this dielectric with a rate dictated by the classical decay length of the amplitude for evanescence, $L_{\text {decay }}$ $=k_{o}^{-1}\left(1 / \sqrt{n_{H}^{2}-n_{\text {eff }}^{2}}\right) \quad\left(k_{o}=2 \pi / \lambda\right.$ is the vacuum wavevector $)$ which can amount to typically $60 \mathrm{~nm}$ at $\lambda=489 \mathrm{~nm}$ for in- termediate index differences $\left(n_{H}-n_{\text {eff }} \sim 0.3-0.5\right)$. The field discontinuity of the $E_{y}$ component in the ratio $\left(n_{H}^{2} / n_{L}^{2}\right)$ further favors a higher field peak value in the gap and minors the relative influence of the high index cylinder field contribution in terms of the mode effective area. Finally, just in the low index gap, the lateral decay is also very quick. One way to grasp the reason why is to note that there is little vertical field decay in this region. Thus the spatial second derivatives needed to ensure the high effective index value in a low index medium are now enforced by this lateral parabolic decay. Beyond this parabolic decay, the field still rapidly evolves (when $x \neq 0$ ) at nearly the same "rate" as the rate that evanescence would adopt vertically in this lowest index medium $n_{L}$ for a tail of any mode with the same effective index. Thus, the key elements are a thin low index gap, and a narrow high-index width for the gap itself but not necessarily for the rest of the high index system, which tends to be ignored thanks to the strong vertical evanescence (itself allowed by some room in the higher index along the vertical, hence somewhat self-consistently but we believe that intuitive pictures are presently of some use).

As for the role of the metal, it may be understood in relation with the two planar plasmonic effective indices $n_{\text {plasm }}^{L, H}=n_{L, H} \times\left[\varepsilon_{\text {metal }} /\left(n_{L, H}^{2}+\varepsilon_{\text {metal }}\right)\right]^{1 / 2}$ (Ref. 1) of the problem, typically $10 \%-20 \%$ higher than corresponding dielectrics indices. For the $L$ plasmonic case reference, it is possible to have an index $n_{\text {eff }}<n_{\text {plasm }}^{L}$ because of the in-plane (lateral) confinement that still lowers the effective index but when enough high index material is added around, $n_{\text {eff }}$ $>n_{\text {plasm }}^{L}$ should rapidly result. For the $H$ plasmonic case reference now, the relation $n_{\text {eff }}<n_{\text {plasm }}^{H}$ should hold unconditionally: the localized role of the $H$ dielectric combined with the lateral confinement both act to lower the index.

Based on this analysis, we propose the geometry of the inverse rib of Fig. 1(b): we first coat a flat plasmonic surface (Au or Ag) with a resist such as poly(methyl methacrylate) (PMMA) or some other low index formulation. Then, lithographically (e.g., by e-beam), we define grooves that are underexposed, so that they adopt some V-shape that stops at a tiny distance from the metal but above it. This delicate point 
can be attained with various steps of additional layers well mastered in resist techniques. Then, we coat the resist and its developed grooves with a high index coating. Such a coating would preferably possess planarizing properties such as those of $\mathrm{BCB}$, ensuring a modest thickness everywhere (say 60-100 nm) but also the formation of an inverse rib, with the desired gap through the low index system at its bottom. To reach higher indices than $\mathrm{BCB}, \mathrm{PVK}$ is a first option but not with a much higher index. High-index waveguides have been most successfully synthesized with tantalum oxide $\mathrm{Ta}_{2} \mathrm{O}_{5}$, which can be only sputtered and whose applicability onto a resist can be questioned. A much safer approach is to use validated sol-gel solutions based on titania $\left(\mathrm{TiO}_{2}\right)$ precursors, which undergo hydrolysis and condensation at modest temperatures, and only demand a final annealing at $150-250{ }^{\circ} \mathrm{C}$ typically. Measurement of modest losses, a few decibel per centimeter, in thin monomode waveguides, ${ }^{22-24}$ have notably demonstrated that the undesirable formation of $\mathrm{TiO}_{2}$ crystallites can be avoided, and scattering can then be limited. With this technique, indices around $n_{H} \sim 2.0$ can be achieved. There are little doubts that wetting properties can be engineered to grant adhesion without air gaps between such sol-gel systems and known resists (PMMA or others).

This inverse rib geometry deserves of course more work to be safely implemented. But it is basically robust against a number of variations. It is clear that our proposal features a remarkable simplicity of fabrication, provided the tiny gap width can be reproducibly obtained: after standard planar coating (say: metal, PMMA), only e-beam lithography with wet etching is required, followed by a planarizing coating. This is at variance with the formation of metallic rib, which usually demands a lift-off step, whose geometric perfection is an issue, since it relies on the cliff-shaped profile of the resist, which entails some longitudinal roughness of said cliff, translating into a wavy profile of the metallic features defined in this way. Great accumulated mastery has reduced this aspect to modest proportions but the intrinsic difficulty of the lift-off approach partly remains. In our proposal, it is also clear that by suppressing the presence of air at the photonic "hot spot" of our system, we guarantee much better aging properties for the metal, either in terms of its adhesive stress $(\mathrm{Au})$ or of its oxidative stress $(\mathrm{Ag})$. This remark is also valid for optically active organic species embedded in one of the coating layers, which are sensitive to oxygen and moist, so that solutions having the metal covered by dielectric can help detecting their interesting interaction with plasmons. ${ }^{25}$

All these favorable properties are true in most variants of our PIROW proposal but what is needed at this first stage is to comfort quantitatively the modal behavior and associated merits. We thus make a simple modeling choice to define a first generation of optimally confined PIROW. Here, our first simulations validate the similarity of the essential ingredients found in the CdS-rod-plasmon-laser geometry: a quick decay and a very strong confinement in the low index gap. Our simulation notably assumes the following parameters: a sidewall off-vertical angle $\theta=15^{\circ}$ ( $75^{\circ}$ slope), a top coating thickness of $\mathrm{e}_{H}=40 \mathrm{~nm}$, a low index coating of $\mathrm{e}_{L}=90 \mathrm{~nm}$, and a gap width d of $10 \mathrm{~nm}$. We use as a continuous param-

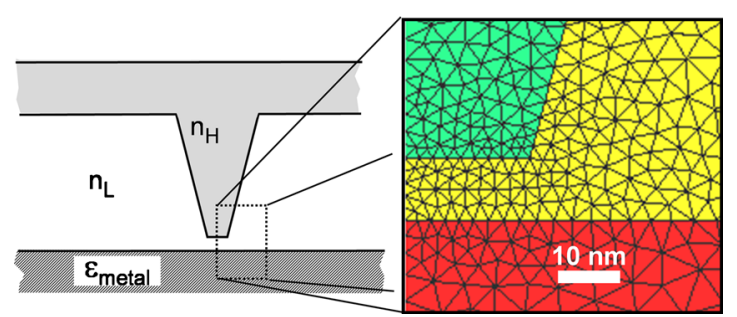

FIG. 2. (Color online) Typical mesh used for the calculation with $d$ $=10 \mathrm{~nm}$ and $L_{\text {tip }}=40 \mathrm{~nm}$.

eter the tip width $L_{\text {tip }}$ of the inverse rib. Subsequently, we will scan the gap size and explore the wavelength dependence of the PIROW.

Technically, we solve the Maxwell spatial differential equations with a finite elements mode solver using hybrid edge/nodal elements as described in Ref. 26. Boundary conditions are defined by perfectly matched layers for anisotropic media, for reflectionless absorption of electromagnetic waves. Figure 2 shows the central part of a typical mesh used for parametric computations presented below. Convergence was checked on finer meshes for selected cases. The gold optical constants, here at $633 \mathrm{~nm}$ but also later in the range $570-850 \mathrm{~nm}$, were interpolated from Johnson and Christy. ${ }^{27}$

\section{SIMULATION RESULTS}

Our first results are concerned with a nearly optimal feasible proposal exploiting sol-gel and, thus, assuming $n_{H}$ $=2.0$. For the resist, there are several possibilities leading to relatively low indices besides the classical PMMA option, thus we stick to $n_{L}=1.4$. We scanned the influence of tip width $L_{\text {tip }}$ [Fig. 1(c)] on the modal behavior to assess the attainment of a confinement similar to that of the plasmonic laser in essence. We start with the value $\mathrm{d}=10 \mathrm{~nm}$. Further below, we study the effect of a different gap width, and next the spectral behavior of the $\mathrm{d}=10 \mathrm{~nm}$ case, including a determination of modal losses. A few tests not reported here have also indicated us that the $\theta=15^{\circ}$ angle is not too far from an optimal system but we intend to extend simulations and deepen our physical understanding of the effect of the angle later.

Figure 3(a) shows a typical color map of the $E_{y}$ field, for the parameter $L_{\text {tip }}=40 \mathrm{~nm}$. The similarity of the field localization with that of Ref. 3 [or Fig. 1(b)] is convincingly achieved, at least qualitatively. To better understand the degree of confinement, with vary $L_{\text {tip }}$ and plot the on-axis vertical $E_{y}(0, y)$ profiles on Fig. 3(b) and the profiles at mid-gap $E_{y}(x, \mathrm{~d} / 2)$ on Fig. $3(\mathrm{c})$ for selected $L_{\text {tip }}$ values as indicated. We plot the effective index on Fig. 3(d) as a function of $L_{\text {tip }}$.

The behavior of the vertical confinement, that is $E_{y}(0, y)$, rapidly reaches its asymptote. It does not decay exponentially in the inverse rib but almost linearly. This is at variance with the case of the nanorod in which the decay seems closer to an exponential shape classically accounted by the length $L_{\text {decay }}=(\lambda / 2 \pi)\left(1 / \sqrt{n_{\text {eff }}^{2}-n_{\text {clad }}^{2}}\right)$ in a cladding of index $n_{\text {clad }}$. The interplay with $x$ confinement still has to be understood to reach future refinements, and improved figures of merit. The decay beyond the rib, in the air above the PIROW, is slower, 

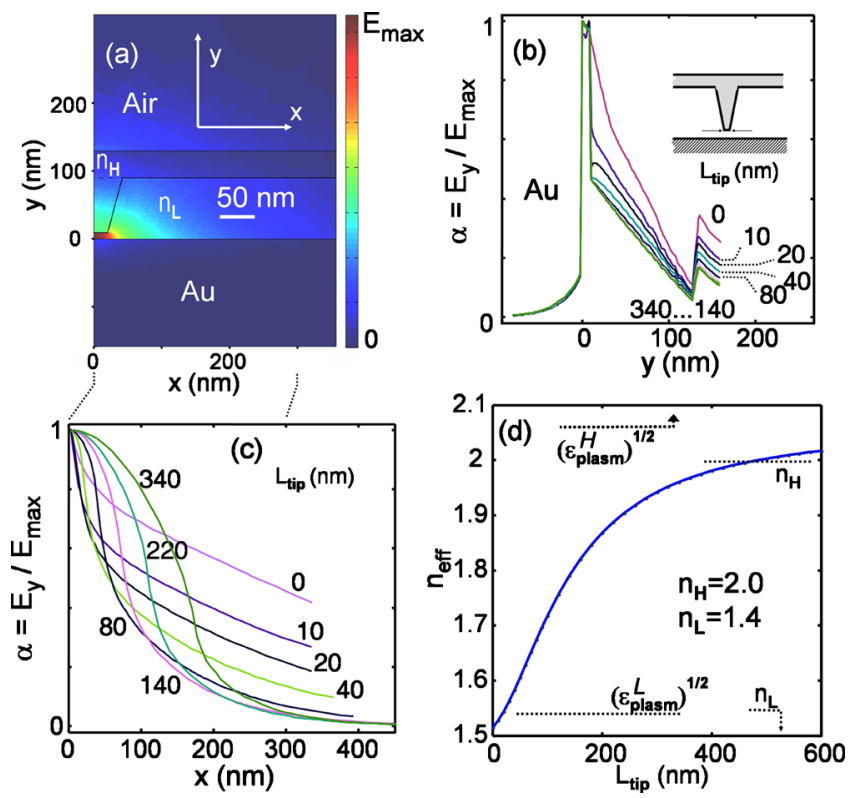

FIG. 3. (Color online) (a) Example of the extremely confined mode of the PIROW for $L_{\text {tip }}=40 \mathrm{~nm}$; (b) field profiles along $y$ at center for selected $L_{\text {tip }}$ values $(0,20,40,80,140,220,340 \mathrm{~nm})$; (c) field profiles along $x$ at height $y=\mathrm{d} / 2$ above metal; (d) PIROW fundamental mode effective index as a function of tip width $L_{\text {tip }}$. Note the evolution with respect to canonical indices of the geometry.

and the field is already very weak, even with the second discontinuity taken into account. This nearly fixed $y$-dependence of the main field components physically means that the $y$ degree of freedom is essentially frozen. As a consequence, the remaining horizontal confinement behaves very much as in a one-dimensional problem for which the well known effective index method could then be applied here to get a simplified view: it starts with a first TM step at successive vertical cuts, to get a profile $n_{\mathrm{eff}}^{\mathrm{TM}}(x)$, which should next be used to solve for the $x$ direction and get an approximate effective index $n_{\text {eff,approx }}^{\mathrm{TE}}$ calculated in the TE polarization.

We further explicit this approach, at least qualitatively, because we found that we can parallel our PIROW results with the well known generic behavior of a square well as regards confinement: this quantity goes through its tightest condition for an intermediate core (well) width. Around this optimum, there is a "deconfined regime" for extremely thin "core." Conversely, in the large width regime, the fundamental mode essentially occupies the growing core volume and swells accordingly.

Data from our calculation do provide a similar trend. We illustrate the confinement trends by looking at the region of $E_{y}$ above a given fraction $\alpha$ of the maximum field $\left|E_{\max }\right|$ as an approximate tool: it is simpler than the exact electromagnetic factors of merit (Poynting vector flux, Purcell factor, etc.) and can be safely used for quick comparison among large data sets. From the two above profiles, $E_{y}(0, y)$ and $E_{y}(x, \mathrm{~d} / 2)$, we determine the width ranges $\Delta x_{\alpha}$ and $\Delta y_{\alpha}$ for which the field obeys $\left|E_{y}\right|>\alpha\left|E_{\max }\right|$. The product $\Delta x_{\alpha} \Delta y_{\alpha}$ defines an area $S_{x y}\left(L_{\text {tip }}, \alpha\right)$ which depends on $L_{\text {tip }}$ and $\alpha$. We then plot $S_{x y}\left(L_{\text {tip }}, \alpha\right)$ as a function of $L_{\text {tip }}$ in Fig. 4.

First, we checked that the discontinuities of $E_{y}(y)$ cause

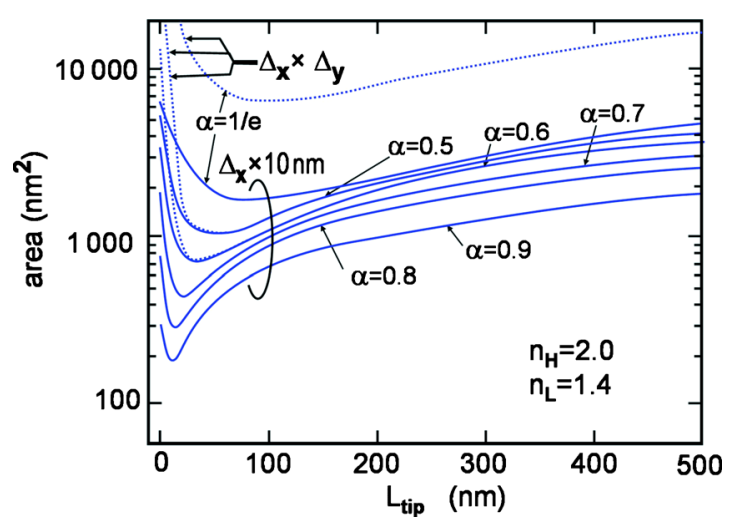

FIG. 4. (Color online) Indicative effective area $S\left(L_{\text {tip }}, \alpha\right)$ taken as $\Delta x \times \Delta y$, the product of ranges along $x$ and $y$ [Figs. 3(b) and 3(c)] for which the normalized field exceeds the indicated threshold $\alpha$ (for $\alpha=1 / e$ and from 0.5 to 0.9 by 0.1 steps). The dotted curves, notably those at the lowest $\alpha$ and $L_{\text {tip }}$ values, are the products $\Delta x \times 10 \mathrm{~nm}, \mathrm{~d}=10 \mathrm{~nm}$ being the gap size within which $E_{y}$ has its peak values.

$\Delta y_{\alpha}$ to coincide with the low index gap region except for the narrowest $L_{\text {tip }}$ values $(20 \mathrm{~nm}$ down to $0 \mathrm{~nm}$ ). For this reason, we plot the gentler curve of $\Delta x_{\alpha} \times 10 \mathrm{~nm}$ as a solid line, and the actual value $S_{x y}\left(L_{\text {tip }}, \alpha\right)$ as a dotted line, thus rendering the main useful trends.

At all threshold $\alpha$ values presented here, the resulting curves $S_{x y}\left(L_{\text {tip }}\right)$ exhibit a minimum between $\sim 10$ and $80 \mathrm{~nm}$. Note that $\alpha$ is a threshold of field amplitude. If the squared field were used, $\alpha=0.7$ would nearly correspond to half-peak intensity and $\alpha=0.6$ to $1 / e$ peak intensity (the Poynting vector flux is more generic than the electric field but its structure is less physically intuitive).

The important variations in the minimum region with $\alpha$ can be understood from the specific shape of $E_{y}(x)$, which tends to display a narrow "nanohat" but a somewhat long tail, the whole profile having thus no fit with basic functions (e.g., Gaussian). At large $\alpha$ values, a narrow area with higher field is present just below the inverse rib tip, and the confinement is markedly better in such an option. But for $\alpha$ $=0.6$ or 0.5 , a flatter minimum is found, at larger tip width $L_{\text {tip. }}$. Different electromagnetic figures of merit apply to define a relevant effective area. Figure 4 indicates how to reconcile possible discrepancies between, e.g., phenomena having an $E^{4}$ dependence (which will tend to be described by the high- $\alpha$ limit $\alpha>0.8$ ) and other phenomena with a simple linear field dependence (which will tend to be described by the low- $\alpha$, say $\alpha \sim 0.5$ limit).

The minimum "area" at $\alpha=0.6$ is $S_{x y}=80 \times 10 \mathrm{~nm}^{2}$, obtained for $L_{\text {tip }} \approx 35 \mathrm{~nm}$. This result compares well with the set of Ref. 3. For example, it compares with their Fig. 1 which indicates $S_{x y}=40 \times 5 \mathrm{~nm}^{2}$ with a similar $\alpha$ criterion, a thinner gap $(5 \mathrm{~nm})$, a shorter wavelength $(489 \mathrm{~nm})$, and a metal of smaller imaginary dielectric constant $(\mathrm{Ag})$.

The comments on the relationship of propagation constants and effective indices to plasmonic ones, made above, may be checked here: the effective index starts below the value of the low index medium $(L) /$ metal plasmon, $n_{\text {eff }}$ $<n_{\text {plasm }}^{L}=1.531$ but can eventually verify $n_{H}<n_{\text {eff }}<n_{\text {plasm }}^{H}$ for a large enough width such as $L_{\text {tip }} \sim 400 \mathrm{~nm}$.

We have checked other cases such as $n_{H}=2.0$ and $n_{L}$ 


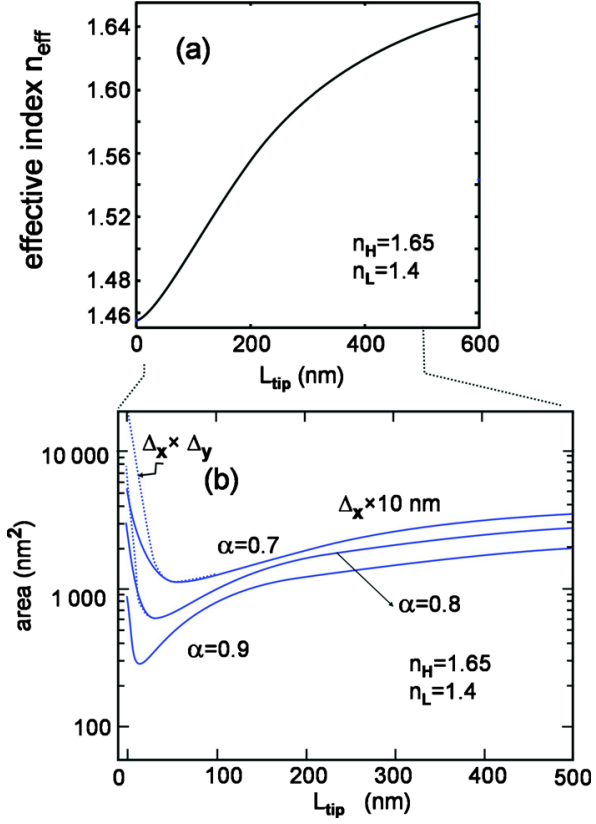

FIG. 5. (Color online) Same as (a) Fig. 3 (effective index) and (b) Fig. 4 (area) for the lower index contrast case configuration as indicated.

$=1.5$ (close to the case of PMMA), and $n_{H}=1.4$ and $n_{L}$ $=1.65$ to address the use of "high index" purely organic resists such as BCB and PVK. They give similar trends. However, strong confinement typically demands an index contrast $n_{H}-n_{L}>0.5$. For a typically "low" index contrast case $\left(n_{H}\right.$ $=1.4$ and $\left.n_{L}=1.65\right)$, lateral $(x)$ confinement looses roughly a factor of 2. Results for this case are shown in Fig. 5(b) in terms of "area," while Fig. 5(a) presents the effective index trend as a function of $L_{\text {tip }}$, with, at $\alpha=0.7$, a typical minimum of $\Delta y=120 \mathrm{~nm}\left(S_{x y}=1200 \mathrm{~nm}^{2}\right) L_{\text {tip }} \sim 50 \mathrm{~nm}$ instead of 54 $\mathrm{nm}$ for the higher contrast case of Fig. 3. Due to the smaller field discontinuity, here, the search for $\Delta y_{\alpha}$ becomes delicate below $\alpha=0.7$.

We now turn to the gap thickness study (Fig. 6). We scan values of d from 4 to $50 \mathrm{~nm}$. The field profiles [Figs. 6(a) and 6(b) ] clearly indicate that the nanohat lateral $(x)$ size is a direct function of d. Localization of the field becomes modest for $\mathrm{d}=20$ to $30 \mathrm{~nm}$, just when d exceeds $L_{\text {tip }}$. Such a scaling is expected for a near-field confinement effect dominated by an electrostatic behavior. In other words the field is dominated by surface charge at the dielectric and metallic interfaces. Figures 6(b) and 6(c) show that, not surprisingly, the effective index diminishes at larger $\mathrm{d}$, favoring a steeper decay in the high index tip. The field inside the gap becomes also less uniform, with the appearance of more explicit cusps typical of interface modes. The confinement evaluation through our simplified area criteria, Fig. 6(d), essentially reflects the 2D character of the situation: For the larger $\alpha$ $=0.7$ value, the area changes by nearly a factor of 100 between $\mathrm{d}=4 \mathrm{~nm}$ and $\mathrm{d}=40 \mathrm{~nm}$, meaning that both vertical and horizontal confinements shrink or swell together. At smaller $\alpha$ values such as $\alpha=0.5$, a transition occurs, when the vertical profile is "aspired" by the tip, changing the $y$ confinement inside the gap. For a more complete account, it would be necessary to investigate the full transition from a dielectric guiding (with the metal remote enough) in the air/H/L structure to the presently studied PIROW limit. For $\alpha=1 / e$, the two variants of our estimate of the confinement are quite distinct. This can be understood since the threshold field along $y$ is located at some height inside the tip $(y>\mathrm{d})$. Still, with this criteria which typically accounts for over $80 \%$ of the modal intensity, the area can be as low as $1000 \mathrm{~nm}^{2}$ for the lower limit of our explored range, $\mathrm{d}=4 \mathrm{~nm}$, translating hence into a spread $\Delta x$ of $250 \mathrm{~nm}$ still around the naive diffraction limit in the low index $\left(\lambda / 2 n_{H}=226 \mathrm{~nm}\right)$.

Overall, our trends indicate a tighter confinement than in Ref. 21, but mostly due to the narrower gap. The rib vs. cylindrical geometry might also favor confinement for our chosen angle.

Our final detailed simulation concerns the spectral behavior of the PIROW (Fig. 7). Here, we use the same parameters as in Figs. 3 and 4 , an angle $\theta=15^{\circ}$ angle and a tip width $L_{\text {tip }}=20 \mathrm{~nm}$, standing above a gap of width $\mathrm{d}$
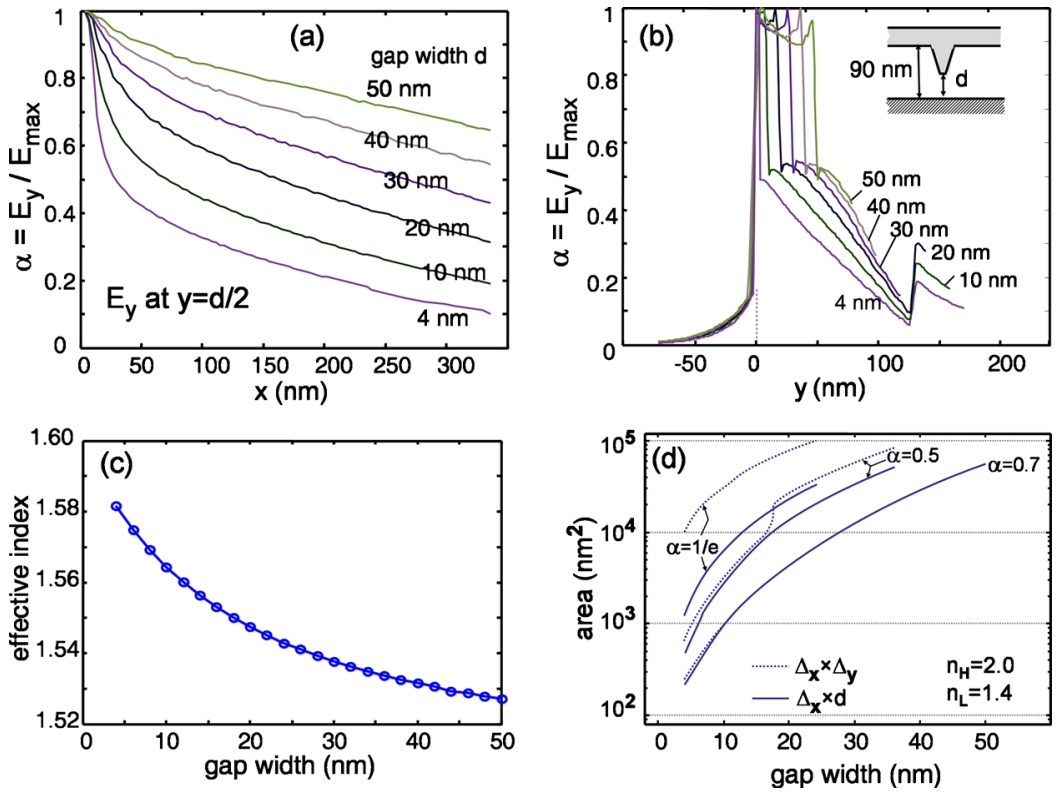

FIG. 6. (Color online) Effect of gap size: (a) normalized field profiles of $E_{y}$ along $x$ at height $y=\mathrm{d} / 2$ above metal for indicated gap values d from 4 to $50 \mathrm{~nm}$; (b) same along $y$, with PIROW scheme in inset; (c) effective index decreases for increasing gap size; (d) indicative effective area $S(\mathrm{~d}, \alpha)$ for $\alpha=0.7, \alpha=0.5$ and $\alpha$ $=1 / e=0.368$, with same variants as in Fig. 4 . 

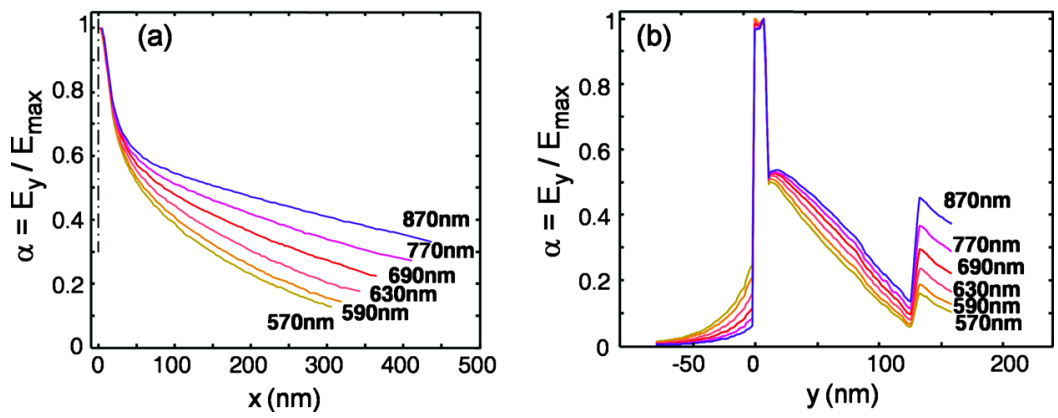

FIG. 7. (Color online) Effect of wavelength on (a) field profile of $E_{y}$ along $x$; (b) field profile of $E_{y}$ along $y$; note that the field pattern under the tip (small $x$ and small positive $y$ ) is unchanged, being thus of quasielectrostatic nature.

$=10 \mathrm{~nm}$ gap and the same index pair $n_{H}=1.4$ and $n_{L}=2.0$. Choosing gold as the metal because of its better technological merit, we expect that on the green-blue side, a plasmonic absorption shall arise. As we can judge from the data of Fig. 7 , the field profiles along $y$, in the gap (the nanohat) and in the tip, are not directly affected by absorption in the 570-850 $\mathrm{nm}$ range investigated here. Rather, we observe that the tails of the $x$ confinement are swelling in the infrared. Similarly, the field in the gap, Fig. 8(b), remains unaffected. The amount of field in the metal increases in on the green side, $\lambda=570 \mathrm{~nm}$, with our normalization but the skin depth decreases, as is usual with plasmons. Because we have chosen a thin layout ( $90 \mathrm{~nm}$ low index $+40 \mathrm{~nm}$ high index), we also have a substantial increase in the field in the air for near infrared wavelength, as the evanescence cannot be sufficiently accommodated in this thin layout. For use in the infrared, if the air/high index interface should play no direct role, a thicker layout should be setup but the risks of either technological difficulties (deeper tip groove before the application of the sol-gel), or of getting into a multimode situation in the high index region, are to be evaluated. Conversely, especially for sensor applications, it could be desirable to use
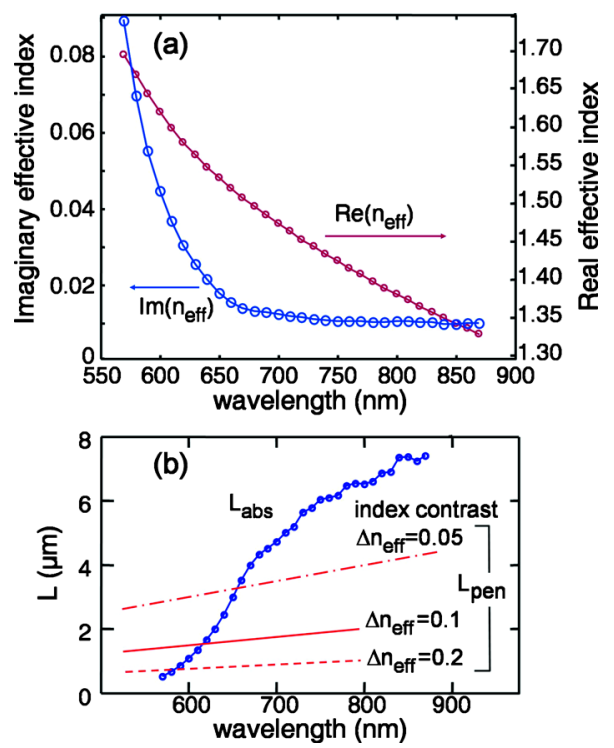

FIG. 8. (Color online) (a) Mode effective index real part, $\operatorname{Re}\left(n_{\text {eff }}\right)$, right scale, and imaginary part $\operatorname{Im}\left(n_{\text {eff }}\right)$, left scale, as a function of vacuum wavelength from orange to near infrared radiations, showing the onset of the gold plasmon below $650 \mathrm{~nm}$; (b) comparison of absorption decay length $L_{\mathrm{abs}}$, with the Bragg penetration depth $L_{\text {pen }}$ for various effective index contrasts $\Delta n_{\text {eff }}$. Bragg reflection survives losses only if $L_{\text {abs }}>L_{\text {pen }}$. the underlying plasmonic properties of the PIROW on the one hand, and a sensing capability of the surface at the nanoscale on the other hand.

The modal properties are also rendered in Fig. 8(a), plotting the real part of the effective index (right scale) and the imaginary part (left scale). The real part evolves between rather large bounds, reflecting the changing capability of the different wavelengths to be confined either close to the tip (green side, $570 \mathrm{~nm}$, large effective index), or to leak appreciably in air (infrared side, $850 \mathrm{~nm}$, low effective index). The assertions made above on the general mechanisms of guidance can be checked against this data set.

The losses are of course of crucial importance. Here, the choice of gold causes a substantial imaginary index, from $\operatorname{Im}\left(n_{\text {eff }}\right)=0.01$ in the far-red to 0.09 in the blue-green for our wavelength range, with a clear onset of the gold band absorption at about $670 \mathrm{~nm}$. Our choice of $633 \mathrm{~nm}$ for the core of the present study thus doubles the far-red penalty, from $\operatorname{Im}\left(n_{\text {eff }}\right) \sim 0.01$ to $\sim 0.02$. In Fig. $8(b)$, we plot the absorption length (power drops by $1 / e$ ), given by $L_{\text {abs }}$ $=\lambda_{\text {vacuum }} /\left[4 \pi \operatorname{Im}\left(n_{\text {eff }}\right)\right]$. Our central choice $\lambda=633 \mathrm{~nm}$ is seen to be at the lower bound of decent propagation length, say $2 \mu \mathrm{m}$. Conversely, lengths above $6 \mu \mathrm{m}$ are easily accessible at little price in terms of confinement. This actually depends how miniature the core of the layout for the targeted application is, as we shall see in Sec. IV, when discussing Bragg reflection. We also note that the propagation length found in Ref. 21, about $10 \mu \mathrm{m}$ at $800 \mathrm{~nm}$ using gold as well, are just slightly larger than ours but their gap is $100 \mathrm{~nm}$.

If a very large gain material is available, the losses may also be offset. ${ }^{3}$ Other techniques that lengthen the propagation length, besides switching from gold to silver, imply the formation of a so-called long-range surface plasmon in a very thin metal layer with symmetric dielectric environment but such low losses are then achieved at the expense of vertical confinement.

\section{APPLICATIONS AND FUNCTIONAL DEVICES}

In view of applications, we now briefly suggest how the PIROW can be adapted to a variety of photonic functions. Such functions are implemented by breaking the translational invariance, as is classical in integrated optics, notably with periodicity. This possibility has notably been exploited with embedded nanorods. ${ }^{28,29}$

With a change in PIROW width $L_{\text {tip }}$ or in PIROW depth, either through a larger e-beam dose exposure, or by careful nanoimprint processes, etc. a periodic pattern can be imple- 
mented so as to cause reflection at a prescribed wavelength $\lambda$. If the width is modulated around some value $L_{\text {tip }}$ [or $\mathrm{W}$ at the top of the inverse rib, as defined Fig. 1(c)], the mean effective index may be used to determine the $\left(a=\lambda / 2 n_{\text {eff }}\right)$ and the modulation $\Delta L_{\text {tip }}$ can be used to reach the desired stop band relative width, assuming $\Delta \lambda / \lambda=(2 / \pi) \Delta n_{\text {eff }} / n_{\text {eff }}$ for square modulation at first Bragg order, $\Delta n_{\text {eff }}$ being the effective index modulation.

If one of the media is active and has gain, a plasmonic laser of distributed feedback (DFB) type can be elaborated much as ${ }^{3}$ and photopumped. The DFB action is a key ingredient for a deterministic-frequency laser.

Let us discuss the feasibility of such Bragg reflectors. It is well known in the physics of DBRs, that in the stop band, the phase varies almost linearly. This means that an equivalent penetration depth $L_{\text {pen }}$ can be defined, such that a DBR acts like a perfect conductor at distance $L_{\text {pen }}$ from its entrance, the periodic arrangement being replaced by the impinging medium index. The formula for $L_{\text {pen }}$ at first order (the so-called quarter-wave stack DBR) is simply $L_{\text {pen }}$ $=\lambda / 4 \Delta n_{\text {eff }}$ because the typical number of layers needed to cause sizable reflection scales like $\langle n\rangle / \Delta n_{\text {eff }},\langle n\rangle$ being an average index, while their physical length scales like $1 /\langle n\rangle$, thus canceling the numerator. Figure 8(b) displays $L_{\text {pen }}$ for various effective index steps $\Delta n_{\text {eff }}$ of $0.05,0.10$, and 0.20 . We have seen that $L_{\text {tip }}$ variations or d variations could easily result in steps of $\Delta n_{\text {eff }} \sim 0.1$, so that a DBR at $\lambda=633 \mathrm{~nm}$ just makes sense. We believe that the similarity of field profiles between the two sections of the DBR period would grant weak extra radiation losses, at least near one edge of the stop band. Also, the attainment of larger index contrasts for weaker $L_{\text {pen }}$ and more favorable $L_{\text {abs }} / L_{\text {pen }}$ ratio might request larger indices from the start, and therefore more opened tip and less steep sidewall, $\theta>15^{\circ}$. Nevertheless, at $\lambda \sim 800 \mathrm{~nm}$ and $\Delta n_{\mathrm{eff}} \sim 0.2$ as suggested by Fig. 3(d) for large tip width modulation, a comfortable ratio $L_{\mathrm{abs}} / L_{\text {pen }}$ $\sim 10$ seems at hand: such a large ratio suggests that reflectivities $>90 \%$ are achievable, be it with the provision of mastered radiation losses.

We briefly elaborate on fabrication. In general, it is delicate to vary for example the inverse rib width without taking the risk of opening the resist down to the metal, which may affect the guiding properties (which should be robustly maintained, however) or cause other unwanted troubles. To circumvent this, a very thin hard-baked layer of resist can be used as the 5-20 $\mathrm{nm}$ bottom layer, on which the sensitive soft-baked layer is further spin-coated. Upon development, the hard-baked bottom layer acts as an etch stop. We intend to detail elsewhere the actual grating geometries to implement various reflectors and in/outcouplers, including concentric outcouplers that transfer energy from a large surface spot to the tiny guiding spot. [The concentric arrangement has been investigated by Radko et al., ${ }^{7}$ quite successfully. Similar concentric devices are investigated by Ref. 30 and were also investigated by companies such as Luxtera, Inc. Ref. 31).] Further possible uses would target nanobiophotonics whereby highly focused waveguides could help addressing biological information into very small volumes. An acoustic analog of largely subwavelength focusing by tapered tips with tight wave confinement was recently given in the hyperlens context. ${ }^{32}$ Highly miniaturized systems can be envisioned notably if they are directly integrated at the pixel scale onto a light-sensitive chip, (e.g., as in Ref. 33).

To finish, we note that our PIROW complements the recently proposed lossless dielectric approaches with similar field profiles but with other fabrication challenges. ${ }^{34}$

\section{CONCLUSION}

The inverse rib geometry with a thin and narrow low index gap between a high index and a plasmonic metal provides a very tight confinement, similar in essence to that of the plasmon laser, which was demonstrated recently. The fabrication simplicity and potential robustness provides this system, which we termed a PIROW, with a capability to serve as a building block for highly miniaturized photonics or also integrating novel plasmonic devices such as lasers. Confinement is clearly obtained down to very small areas of less than $\sim 500 \mathrm{~nm}^{2}$ without the need to etch the metal. The critical metal integrity and perfection are thus kept maximal. The capability of sol-gel layered synthesized at lowtemperature to serve into low loss optical waveguides by simple spin coating further supports our proposal. We have provided the main basic items needed to build up devices: effective indices (in the visible) and modal areas, for selected cases. We notably addressed the role of the two small parameters, the tip width and the gap width. The level of losses arising from the gold layer was described as a function of wavelength. Gold incurs an imaginary index between 0.01 on the far-red side $(800 \mathrm{~nm})$ and 0.1 at the orange side $(570$ $\mathrm{nm}$ ) of our scan. This is still sizable but it could be obviated by mitigating the plasmonic confinement with some extra dielectric confinement or by using silver.

\section{ACKNOWLEDGMENTS}

We acknowledge the help of Emmanuel Rousseau and suggestions of J.-J Greffet and T. Gacoin.

${ }^{1}$ V. Z. Zayats, I. I. Smolyaninov, and A. A. Maradudin, Phys. Rep. 408, 131 (2005).

${ }^{2}$ S. A. Maier, Plasmonics: Fundamentals and Applications (Springer, Berlin, 2007), Vol. XXV.

${ }^{3}$ R. F. Oulton, V. J. Sorger, and T. Zentgraf, Nature (London) 461, 629 (2009).

${ }^{4}$ P. Berini, Phys. Rev. B 63, 125417 (2001).

${ }^{5}$ P. Berini, Phys. Rev. B 61, 10484 (2000).

${ }^{6}$ M. L. Nesterov, D. Martin-Cano, A. I. Fernandez-Dominguez, E. Moreno, and F. J. Garcia-Vidal, Opt. Lett. 35, 423 (2010).

${ }^{7}$ I. P. Radko, S. I. Bozhevolnyi, G. Brucoli, L. Martin-Moreno, F. J. GarciaVidal, and A. Boltasseva, Opt. Express 17, 7228 (2009).

${ }^{8}$ J. Grandidier, S. Massenot, G. Colas des Francs, A. Bouhelier, J.-C. Weeber, L. Markey, A. Dereux, J. Renger, M. U. Gonzalez, and R. Quidant, Phys. Rev. B 78, 245419 (2008).

${ }^{9}$ E. J. R. Vesseur, R. De Waele, H. J. Lezec, H. A. Atwater, F. J. Garcia de Abajo, and A. Polman, Appl. Phys. Lett. 92, 083110 (2008).

${ }^{10}$ A. Boltasseva, V. S. Volkov, R. B. Nielsen, E. Moreno, S. G. Rodrigo, and S. I. Bozhevolnyi, Opt. Express 16, 5252 (2008).

${ }^{11}$ T. Holmgaard and S. I. Bozhevolnyi, Phys. Rev. B 75, 245405 (2007).

${ }^{12}$ K. Leosson, T. Nikolajsen, A. Boltasseva, and S. I. Bozhevolnyi, Opt. Express 14, 314 (2006).

${ }^{13}$ J. A. Dionne, L. A. Sweatlock, H. A. Atwater, and A. Polman, Phys. Rev. B 73, 035407 (2006).

${ }^{14}$ T. Søndergaard, S. I. Bozhevolnyi, and A. Boltasseva, Phys. Rev. B 73, 
045320 (2006)

${ }^{15}$ S. I. Bozhevolnyi, V. S. Volkov, E. Devaux, and T. W. Ebbesen, Phys. Rev. Lett. 95, 046802 (2005).

${ }^{16}$ A. Boltasseva, S. I. Bozhevolnyi, T. Sondergaard, T. Nikolajsen, and K. Leosson, Opt. Express 13, 4237 (2005).

${ }^{17}$ J. A. Dionne, L. A. Sweatlock, H. A. Atwater, and A. Polman, Phys. Rev. B 72, 075405 (2005)

${ }^{18}$ S. A. Maier and H. A. Atwater, J. Appl. Phys. 98, 011101 (2005).

${ }^{19}$ S. I. Bozhevolnyi, J. Erland, K. Leosson, P. M. W. Skovgaard, and J. M. Hvam, Phys. Rev. Lett. 86, 3008 (2001).

${ }^{20}$ I. De Leon and P. Berini, Phys. Rev. B 78, 161401 (2008).

${ }^{21}$ S. I. Bozhevolnyi and K. V. Nerkararyan, Opt. Express 17, 10327 (2009).

${ }^{22}$ M. Bedu, G. Sagarzazu, T. Gacoin, P. Audebert, C. Weisbuch, and L. Martinelli, "Sol-gel planar waveguides for improved fluorescence microarrays," Thin Solid Films 518, 4450 (2010).

${ }^{23}$ G. Sagarzazu, M. Bedu, L. Martinelli, K.-N. Ha, N. Pelletier, V. I. Safarov, C. Weisbuch, T. Gacoin, and H. Benisty, SPIE Photonics Europe, Biophotonics: Photonic Solutions for Better Health Care, Strasbourg, France, 29 May 2008.
${ }^{24}$ G. Sagarzazu, M. Bedu, L. Martinelli, N. Pelletier, V. I. Safarov, C. Weisbuch, T. Gacoin, and H. Benisty, Biosens. Bioelectron. 24, 2281 (2009).

${ }^{25}$ J. Bellessa, C. Bonnand, J. C. Plenet, and J. Mugnier, Phys. Rev. Lett. 93, 036404 (2004).

${ }^{26}$ J. Jin, The Finite Element Method in Electromagnetics (Wiley, New York, 1993).

${ }^{27}$ P. B. Johnson and R. W. Christy, Phys. Rev. B 6, 4370 (1972).

${ }^{28}$ Y. Zhang and M. Loncar, Opt. Express 16, 17400 (2008).

${ }^{29}$ C. J. Barrelet, Nano Lett. 6, 11 (2006).

${ }^{30}$ F. Van Laere, T. Claes, J. Schrauwen, S. Scheerlinck, W. Bogaerts, D. Taillaert, L. O'Faolain, D. Van Thourhout, and R. Baets, IEEE Photon. Technol. Lett. 19, 1919 (2007).

${ }^{31}$ L. C. Gunn, III, T. J. Pinguet, M. J. Rattier, and J. Witzens, "Polarization splitting grating couplers," U.S. Patent No. 7,068,887 B1 (2006).

${ }^{32}$ J. Li, L. Fok, X. Yin, G. Bartal, and X. Zhang, Nature Mater. 8, 931 (2009).

${ }^{33}$ L. Martinelli, H. Choumane, N.-K. Ha, G. Sagarzazu, C. Goutel, and C. Weisbuch, Appl. Phys. Lett. 91, 083901 (2007).

${ }^{34}$ M. Davanço and K. Srinivasan, Opt. Express 18, 10995 (2010). 